\title{
Simulation of Indian summer monsoon rainfall, interannual variability and teleconnections: Evaluation of CMIP6 models
}

Kavirajan Rajendran ( $\sim$ rajend@csir4pi.in )

CSIR Fourth Paradigm Institute https://orcid.org/0000-0002-9437-4729

\section{Sajani Surendran}

CSIR Fourth Paradigm Institute

\section{Stella Jes Varghese}

AcSIR: Academy of Scientific and Innovative Research

Anjali Sathyanath

CSIR Fourth Paradigm Institute

\section{Research Article}

Keywords: Indian summer monsoon rainfall, Interannual variability, CMIP6, monsoon teleconnections, ENSO, 24 EQUINOO

Posted Date: February 17th, 2021

DOl: https://doi.org/10.21203/rs.3.rs-242562/v1

License: (9) This work is licensed under a Creative Commons Attribution 4.0 International License. Read Full License 


\section{Abstract}

We analyse the performance of global climate models of 6th generation of Coupled Model Intercomparison Project (CMIP6) in simulating climatological summer monsoon rainfall over India, interannual variability (IAV) of all-India summer monsoon rainfall (ISMR) and its teleconnections with rainfall variability over equatorial Pacific and Indian Oceans. The multimodel ensemble mean (MME) of 61 CMIP6 models shows the best skill in simulating mean monsoon rainfall over India compared to the MMEs of 6th generation atmosphere-only models (AMIP6) and the previous generations of Atmospheric and Coupled Model Intercomparison Projects (AMIPs and CMIPs). Systematic improvement and reduction in bias are evident from lower to higher AMIPs/CMIPs. Still, there exists dry bias over a narrow region of the monsoon zone of central India besides wet and cold bias over the surrounding oceans. The persistence of errors in atmosphere-only models hints that the source of errors could be with atmosphere models. Fifteen CMIP6 models selected through objective criteria, perform the best in simulating mean monsoon, IAV of ISMR, the strong inverse relationship between ISMR and Boreal summer El NinoSouthern Oscillation (ENSO), and the inverse relationship between all-India rainfall and north-west tropical Pacific rainfall in June. Several models reproduce the dipole structure of Equatorial Indian Ocean Oscillation (EQUINOO) with the centres over western and eastern equatorial Indian Ocean. But, ISMREQUINOO relationship in many of them is opposite to the observed. Our analysis implies the need for capturing ISMR-EQUINOO link to improve the simulation of IAV of ISMR which is crucial for reliable monsoon prediction and projection.

\section{Full Text}

Due to technical limitations, full-text HTML conversion of this manuscript could not be completed. However, the manuscript can be downloaded and accessed as a PDF.

\section{Figures}




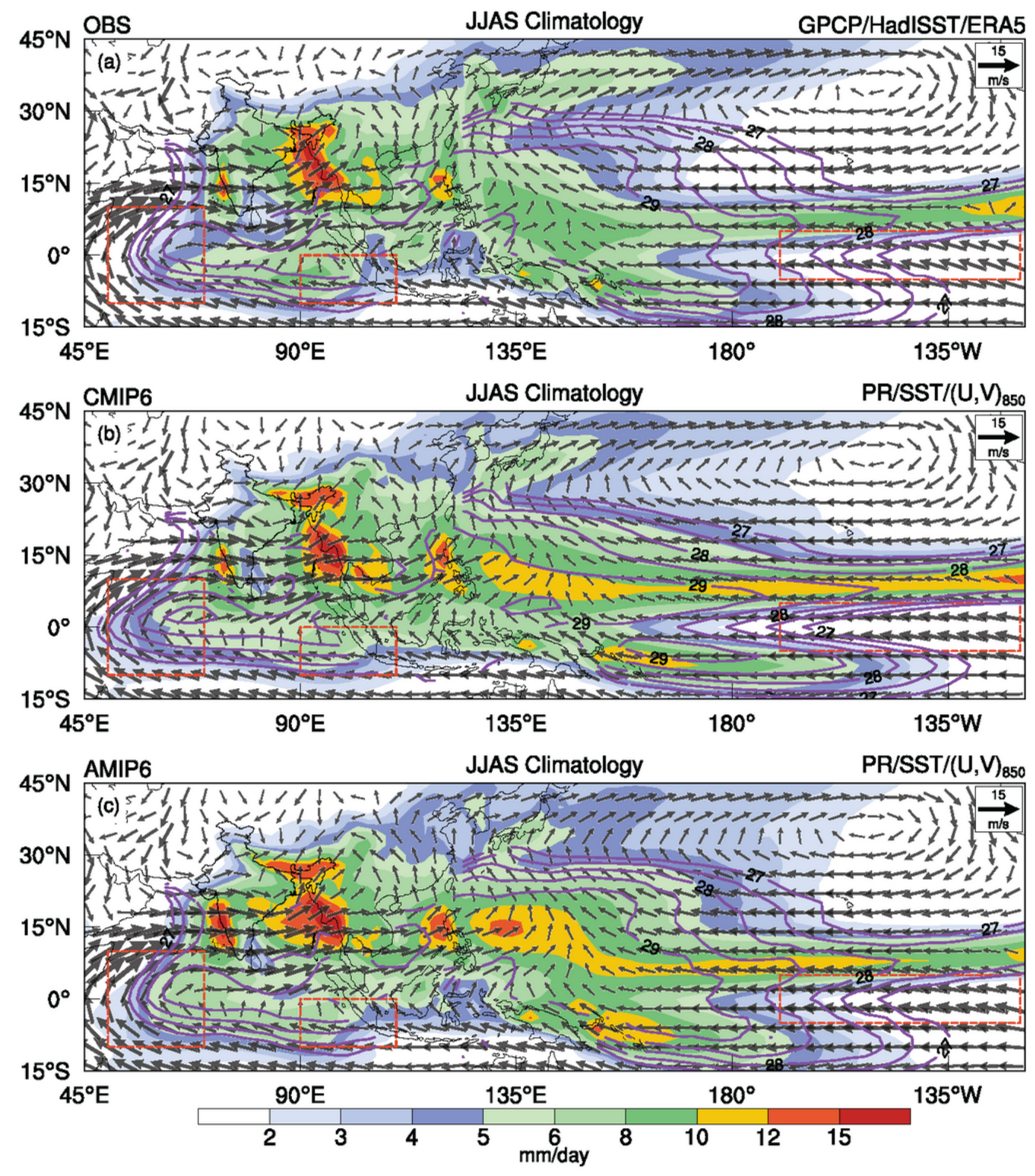

Figure 1

Climatological JJAS mean rainfall (shaded), SST (purple contours) and $850 \mathrm{hPa}$ wind (vectors) from the observations (top panel) and the MMEs of models of CMIP6 (middle panel) and AMIP6 (bottom panel). Red solid boxes indicate regions of western and eastern equatorial Indian Ocean (WEIO and EEIO respectively) and equatorial central Pacific (NINO3.4). 


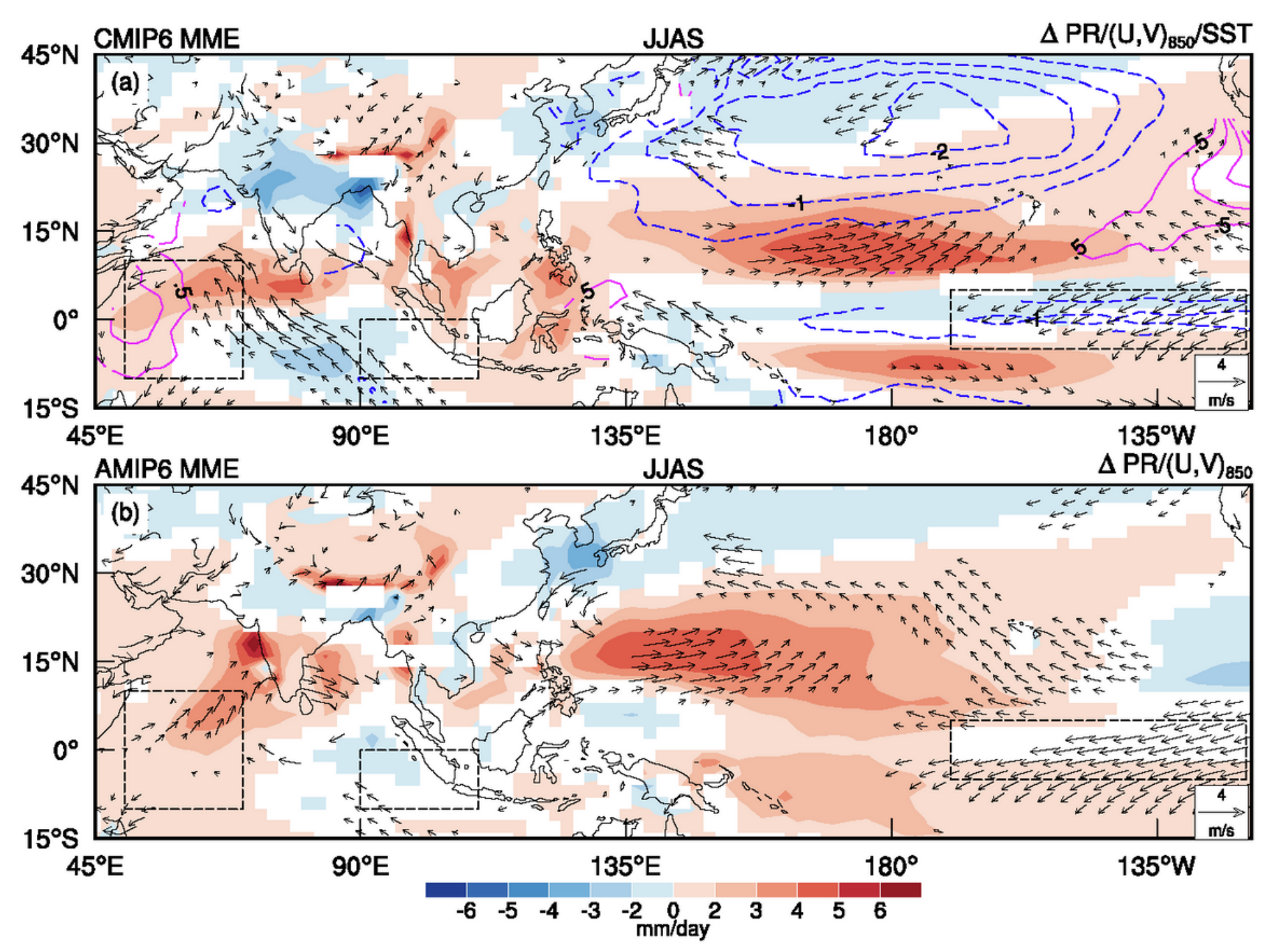

Figure 2

Differences in climatological JJAS mean rainfall (shaded) and $850 \mathrm{hPa}$ wind (vectors) in the MMEs of models of CMIP6 (top panel) and AMIP6 (bottom panel) from the corresponding observations. For the MME of CMIP6 models, positive (negative) SST biases are shown in purple solid (blue dashed) contours in the top panel. Climatological biases significant at $95 \%$ level are shown. Black dashed boxes indicate regions of western and eastern equatorial Indian Ocean (WEIO and EEIO respectively) and equatorial central Pacific (NINO3.4). 

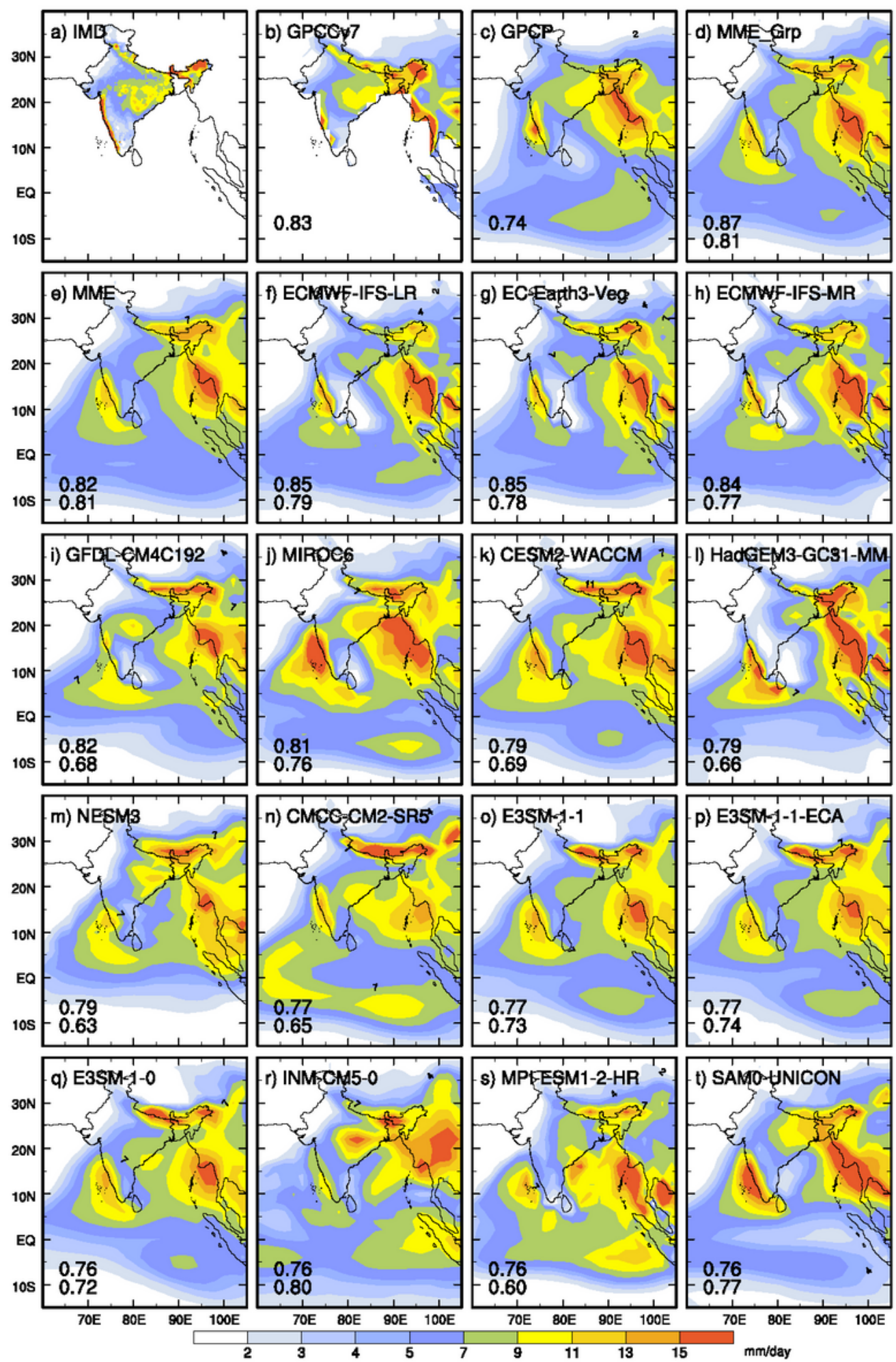

Figure 3

a-d). Spatial distribution of climatological JJAS mean rainfall from observations of a) IMD, b) GPCC v7 and c) GPCP, and d) theMMEof 15 CMIP6 models (MME Grp) selected as the best group for ISMR simulation (CMIP6 Grp) (left to right in top row. e-t). The corresponding distributions from the MME of all 61 CMIP6 models and 15 members of CMIP6 Grp. The pattern correlations PCCIMD (PCCGPCP) with IMD (GPCP) rainfall over India (the extended Indian region) for simulations are given as the first (second) 


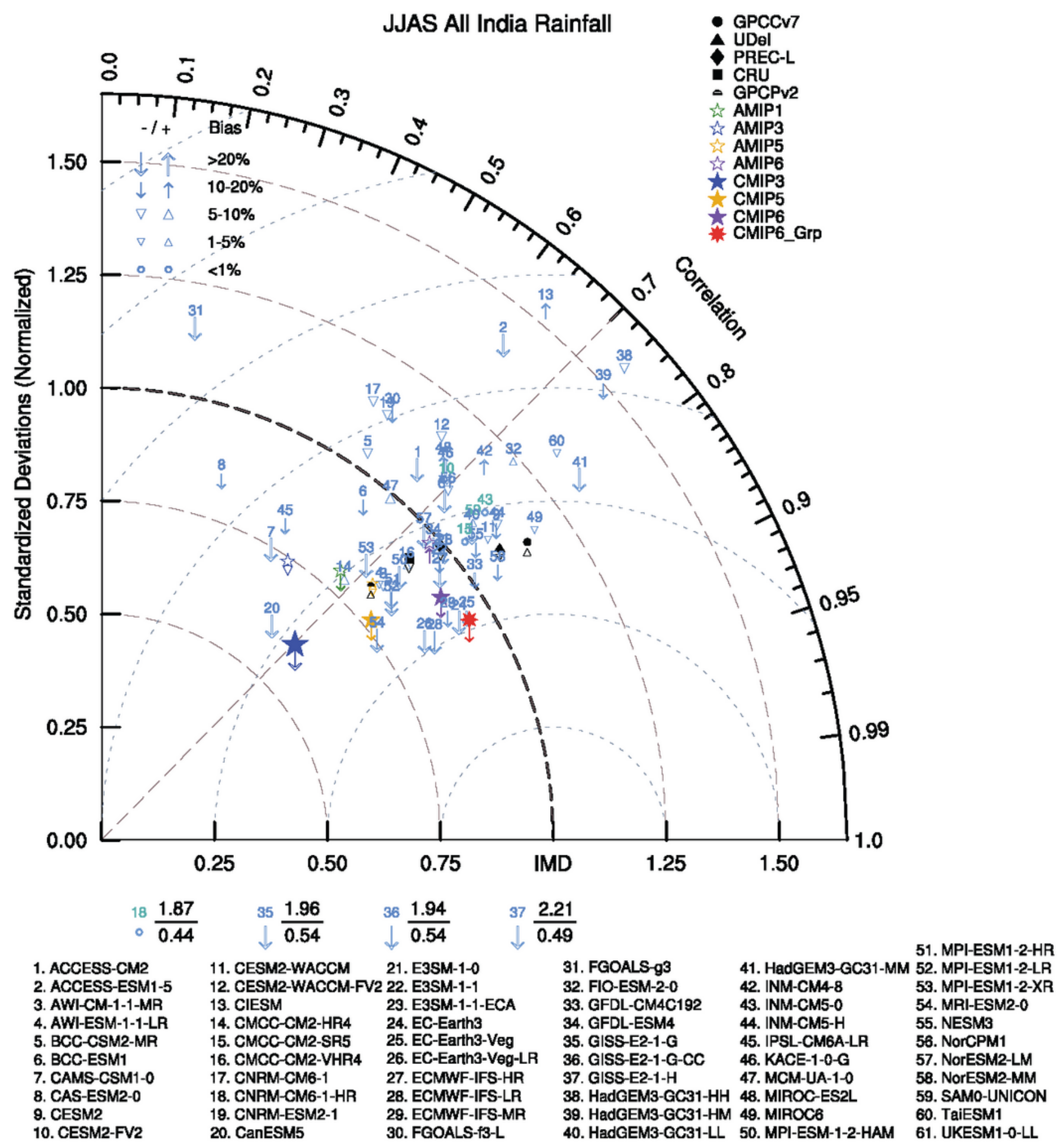

Figure 4

Taylor diagram comparing the statistics from 61 CMIP6 models, 5 observation datasets of rainfall (GPccv7, UDel, PREC-L, CRU and GPCPv2 denoted by black filled symbols), the MME of 15 CMIP6 models (MME Grp) with better skill in simulating ISMR climatology (CMIP6 Grp) and the MMEs of models of 
CMIP6, AMIP6, CMIP5, AMIP5, CMIP3, AMIP3 and AMIP1. Statistics plotted are i) pattern correlation coefficient (PCC) given by the azimuthal angle, normalised standard deviation as the radial distance and the normalised standard deviation of errors as semicircles centred around the IMD label. The lower symbol attached to each symbol shows the BIAS in simulating the mean rainfall with respect to IMD observation.
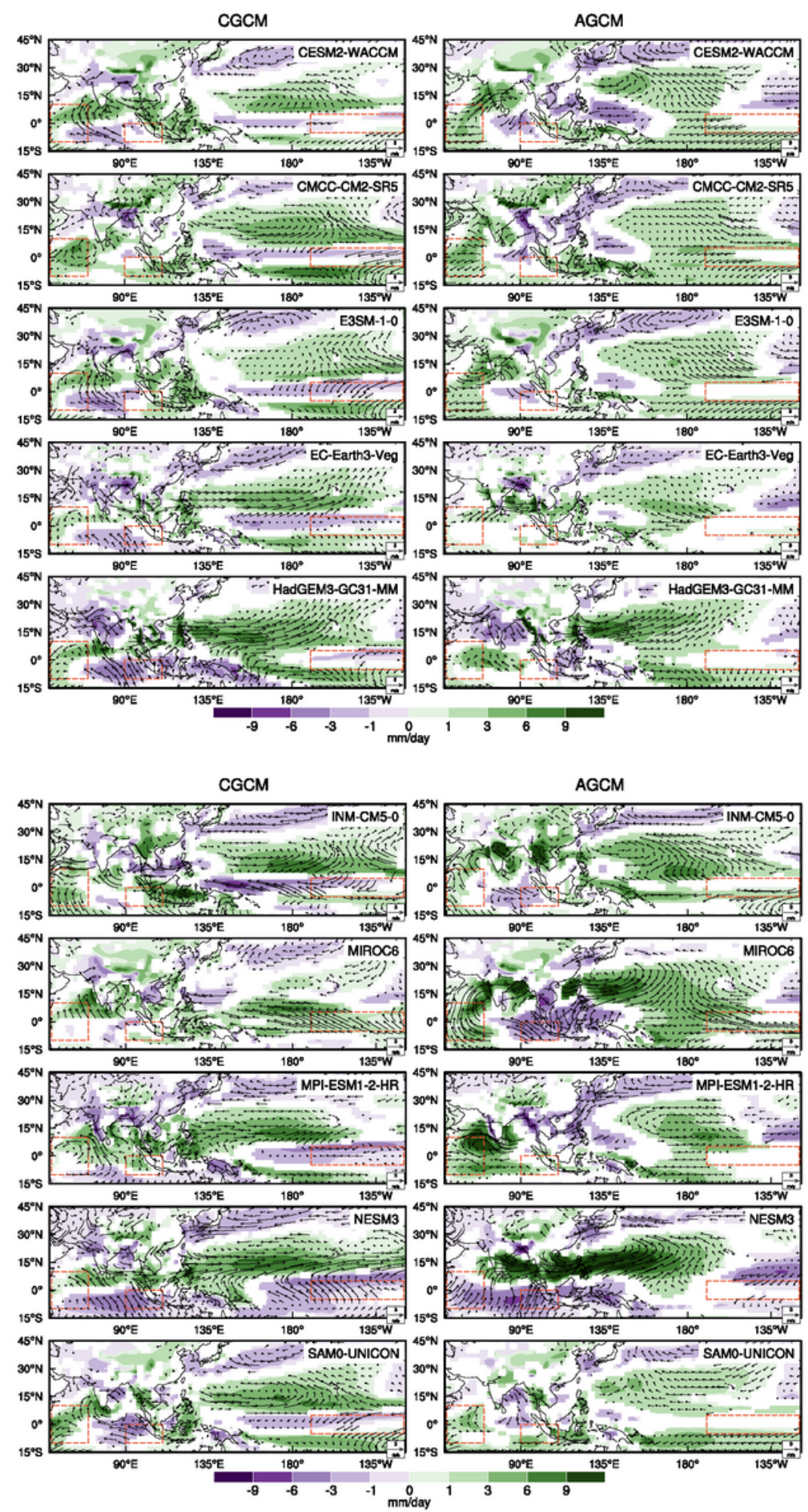

Figure 5 
Differences in climatological JJAS mean rainfall (shaded) and $850 \mathrm{hPa}$ wind (vectors) in the coupled models (left panels) and their atmosphere-only models (right panels) available for 10 models of the selected group of CMIP6 models (CMIP6 Grp). Climatological biases significant at 90\% level are shown. Red dashed boxes indicate regions of western and eastern equatorial Indian Ocean (WEIO and EEIO respectively) and equatorial central Pacific (NINO3.4).
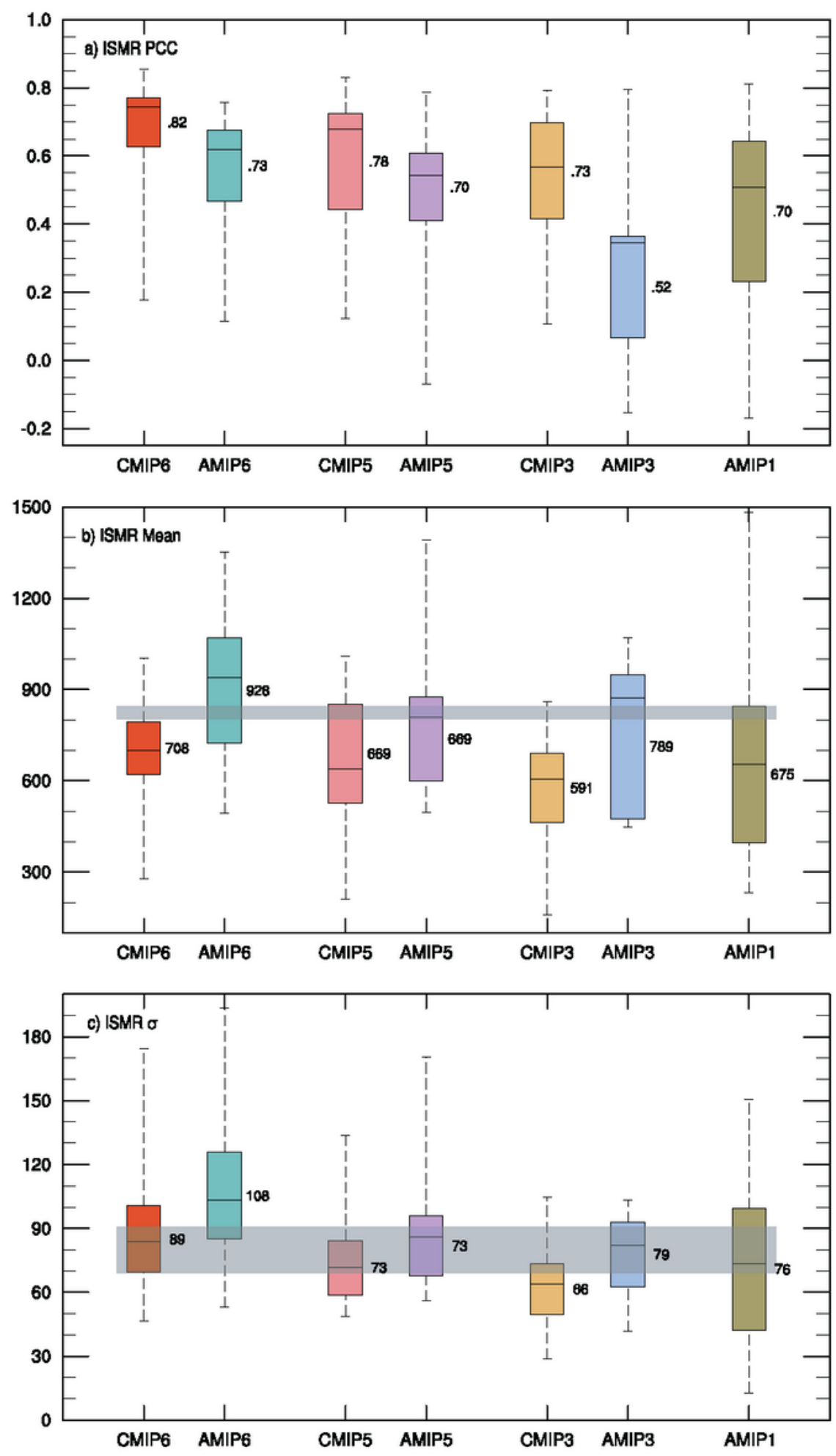

Figure 6 
a) Box and whisker plots showing the PCCs of mean summer monsoon rainfall over India against the corresponding IMD observation for CMIP6, AMIP6, CMIP5, AMIP5, CMIP3, AMIP3 and AMIP1 models. Whiskers show the maximum and minimum values among the PCCs of the member models and the box shows the interquartile range or the first to the third quartile (second quartile or the median is shown as the solid line inside the box) of the PCCs (top panel). The PCC for the MME of models from each MIP is written in the right side of its box. b) Same as a), but for all-India total summer monsoon rainfall $(\mathrm{m})$ in $\mathrm{mm}$ (middle panel). The range of observed $\mathrm{m}$ estimated for the periods corresponding to different MIPs are shown in the background as grey shaded box. c) Same as b), but for the standard deviation of allIndia summer monsoon rainfall in $\mathrm{mm}$ (bottom panel). 

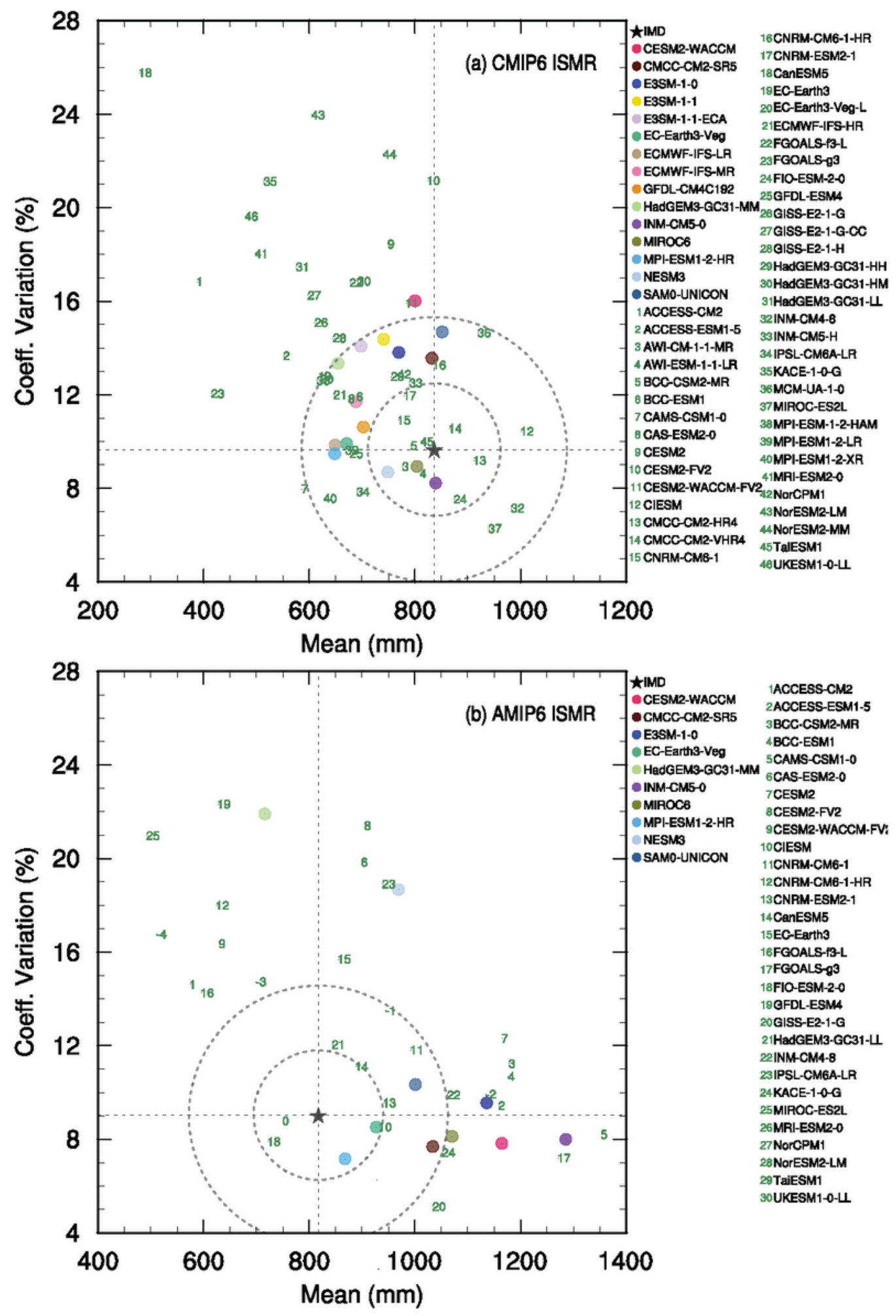

Figure 7

Climatological all-India total summer monsoon rainfall ( $\mathrm{m}$ in $\mathrm{mm}$ ) versus its interannual coefficient of variation (standard deviation divided by $\mathrm{m}$ as \%) in i) CMIP6 (top panel) and ii) AMIP6 (bottom panel) simulations. Observed mean and CV from IMD rainfall over the respective base periods of 1951-2010 and 1979-2010 respectively, are denoted by the black star. Two dashed circles around the observations are of radii $₫ 15 \%$ (inner) and $₫ 30 \%$ of the observations. 

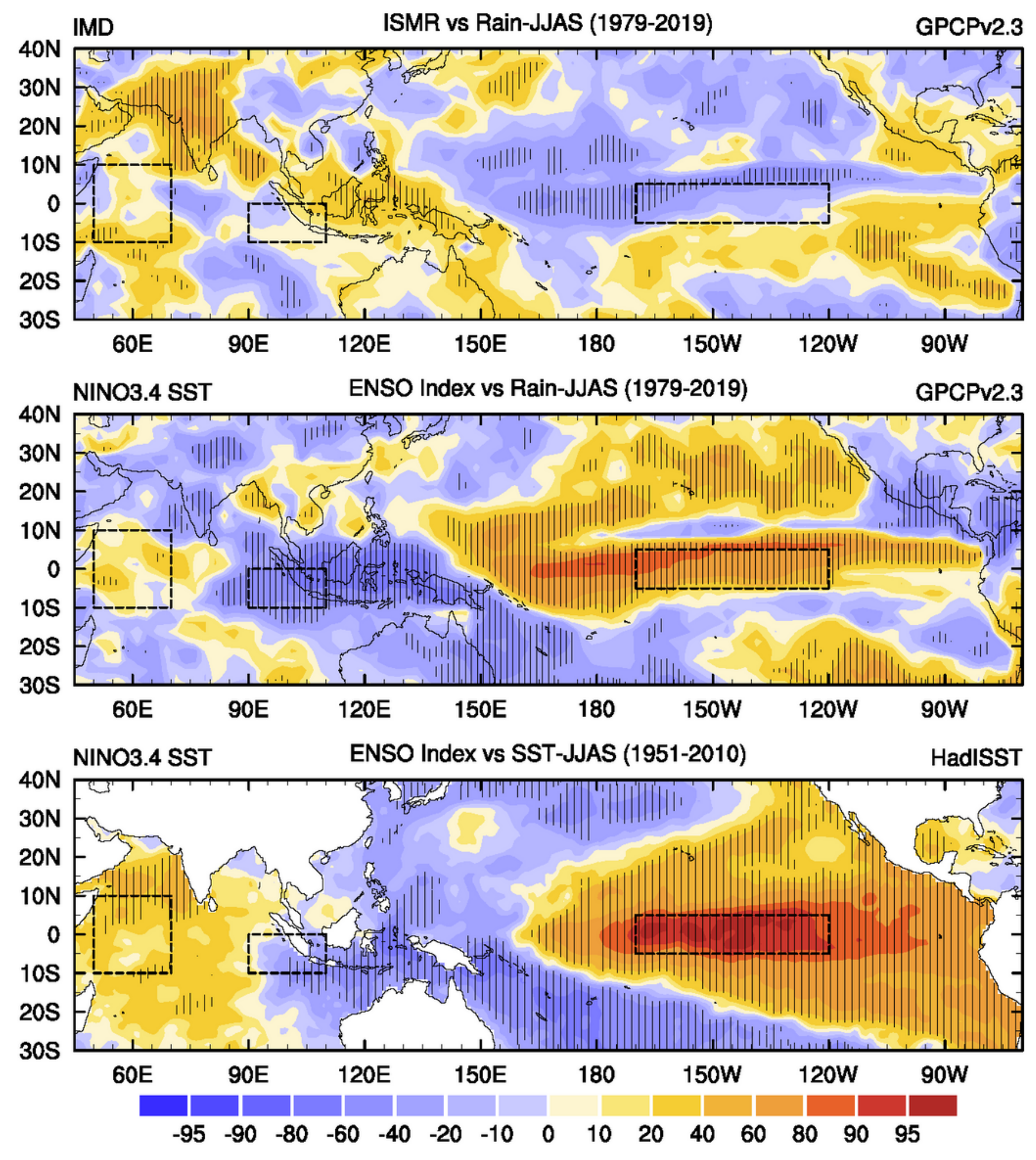

Figure 8

Distribution of correlation coefficient of a) ISMR or all-India average summer monsoon rainfall versus rainfall at every grid over the tropical Asia-Pacific sector (top panel), b) ENSO index versus rainfall at every grid over the tropical Asia-Pacific sector (middle panel) and c) ENSO index versus SST at every grid over the tropical Asia-Pacific sector (bottom panel). Black dashed boxes show regions of western and 
eastern equatorial Indian Ocean (WEIO and EEIO respectively) and equatorial central Pacific (NINO3.4). Correlation coefficients significant at $95 \%$ are stippled.

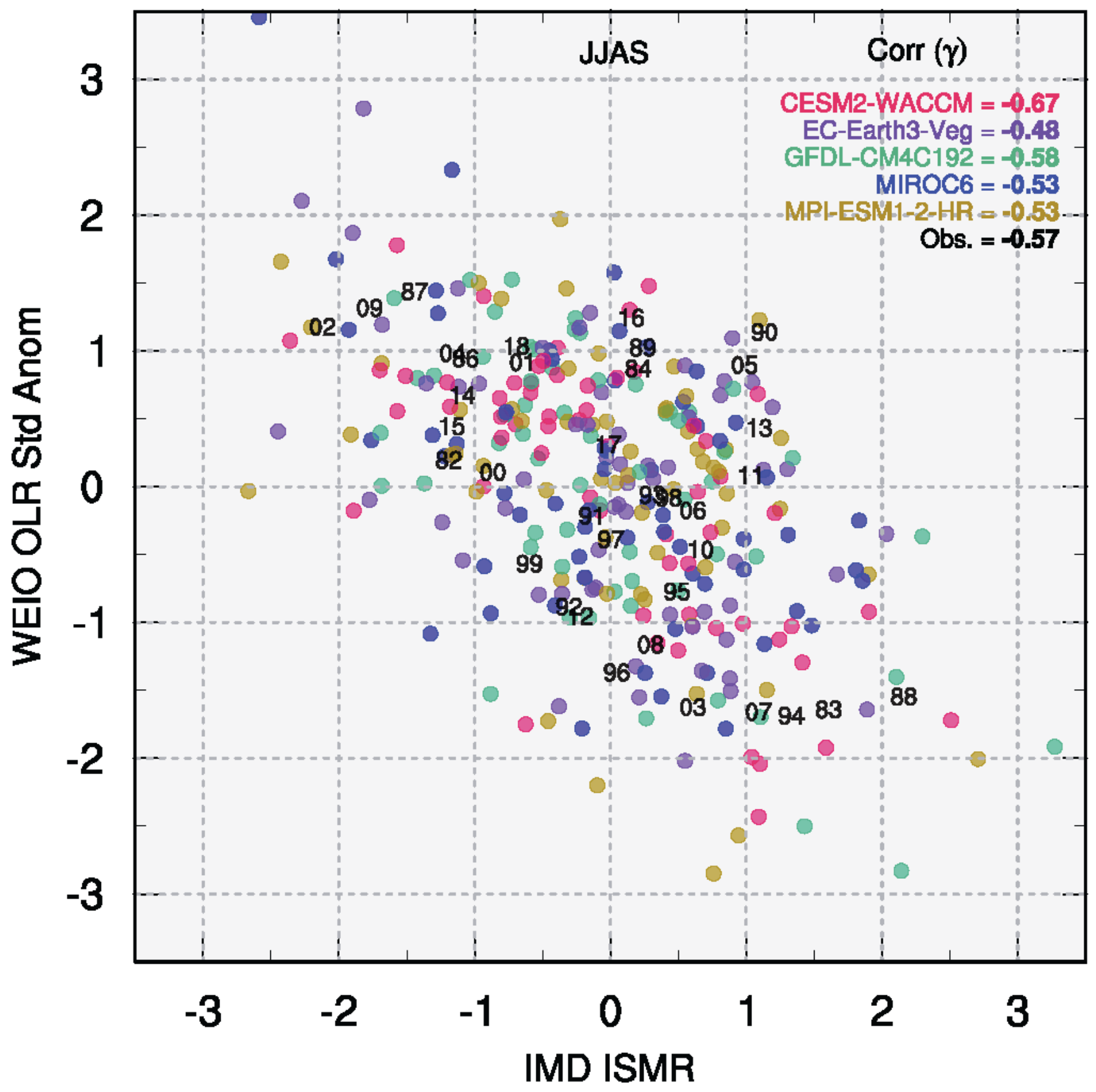

Figure 9

Scatter plot of standardised anomalies of ISMR versus WEIO OLR from observation (IMD rainfall and NOAA CDR denoted by the last two digits of the years) and 5 representative models of CMIP6 Grp. The corresponding correlations are given in top-right corner. 

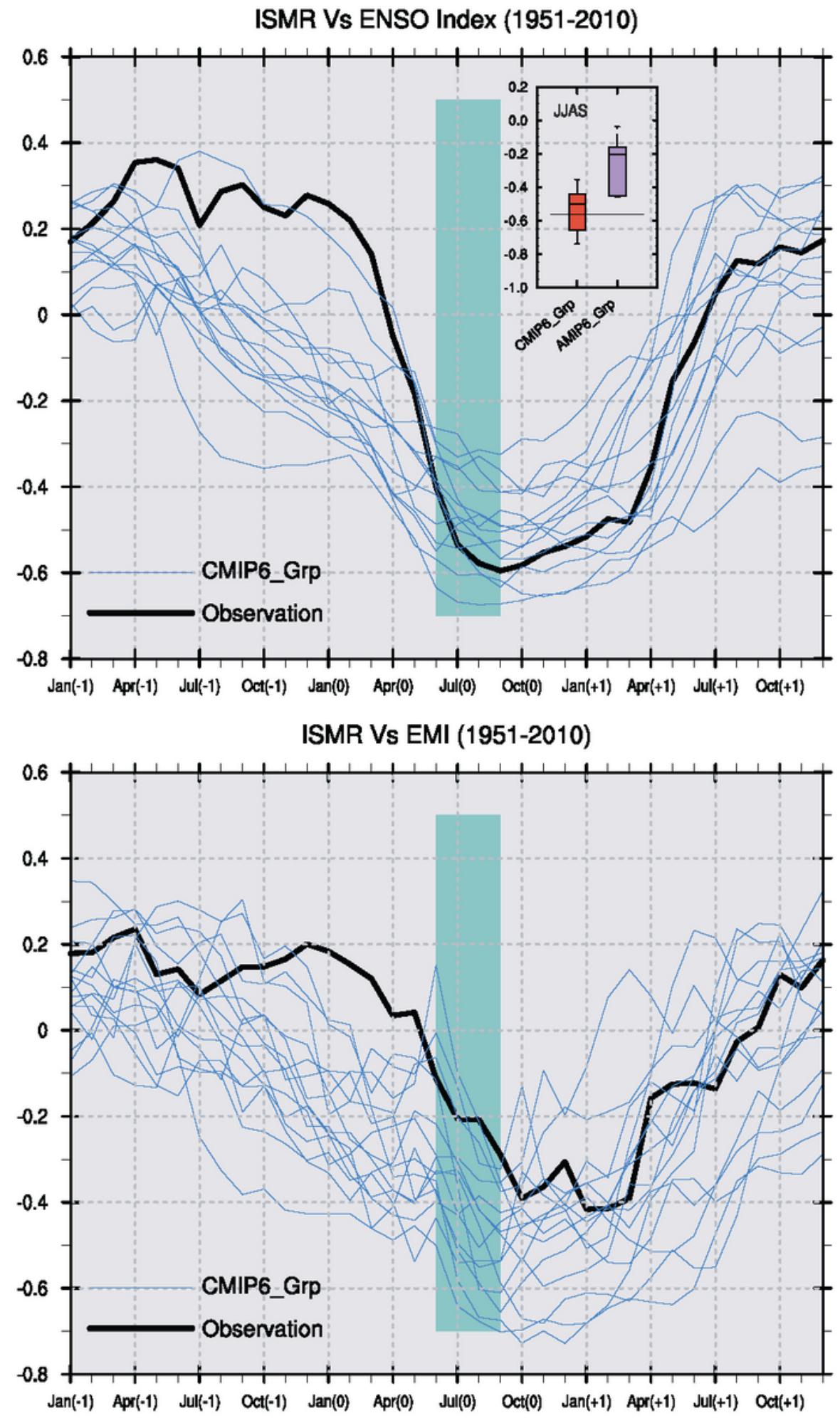

\section{Figure 10}

a) Correlation coefficients between Indian summer monsoon rainfall (ISMR) anomaly and NINO3.4 SST anomaly (ENSO index) at various monthly lags for observation (black thick line) and 15 models (blue thin lines) of CMIP6 Grp (top panel). JJAS season is highlighted in green. Top panel inset: Box and whisker plots showing the correlation coefficient between ISMR and ENSO index for 15 CMIP6 Grp models and 10 of their corresponding atmosphere-only models from AMIP6. Whiskers show the maximum and minimum 
values among the correlations of the member models and the box shows the interquartile range or the first to the third quartile (second quartile or the median is shown as the solid line inside the box). The horizontal black line denotes the observed correlation of -0.56. b) Same as a), but for correlation coefficients between ISMR anomaly and El Ni no Modoki Index (EMI) at various monthly lags (bottom panel).

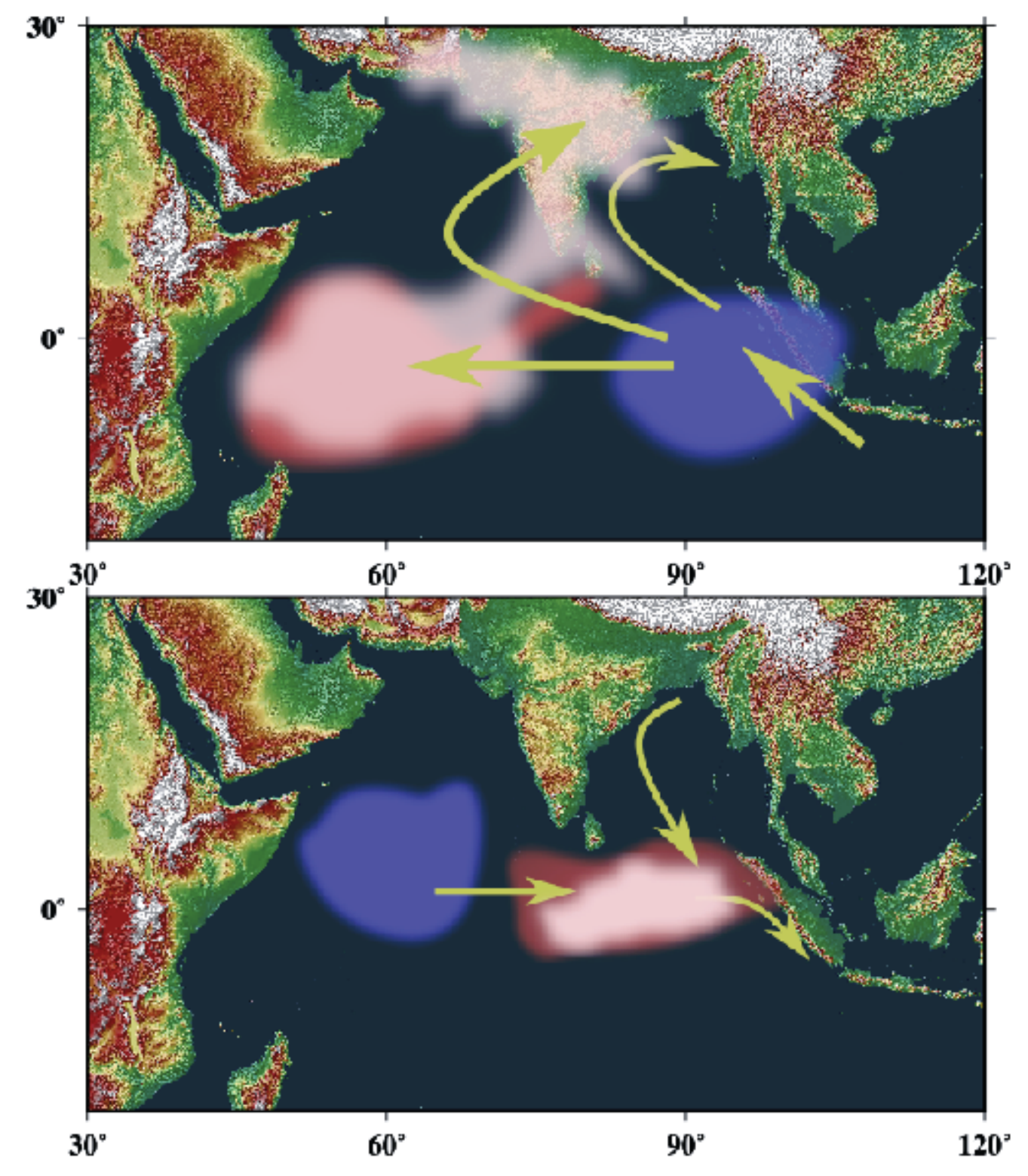

\section{Figure 11}

Schematic diagrams showing positive (top panel) and negative (bottom panel) phases of Equatorial Indian Ocean Oscillation (EQUINOO). The anomalies of SST (red: warmer \& blue: colder), direction of prevalent surface winds (yellow arrows) and cloudiness (silver shade) as proxy for organized convection/rainfall associated with the two phases, are also shown. Note: The designations employed and the presentation of the material on this map do not imply the expression of any opinion whatsoever on the part of Research Square concerning the legal status of any country, territory, city or area or of its 
authorities, or concerning the delimitation of its frontiers or boundaries. This map has been provided by the authors.

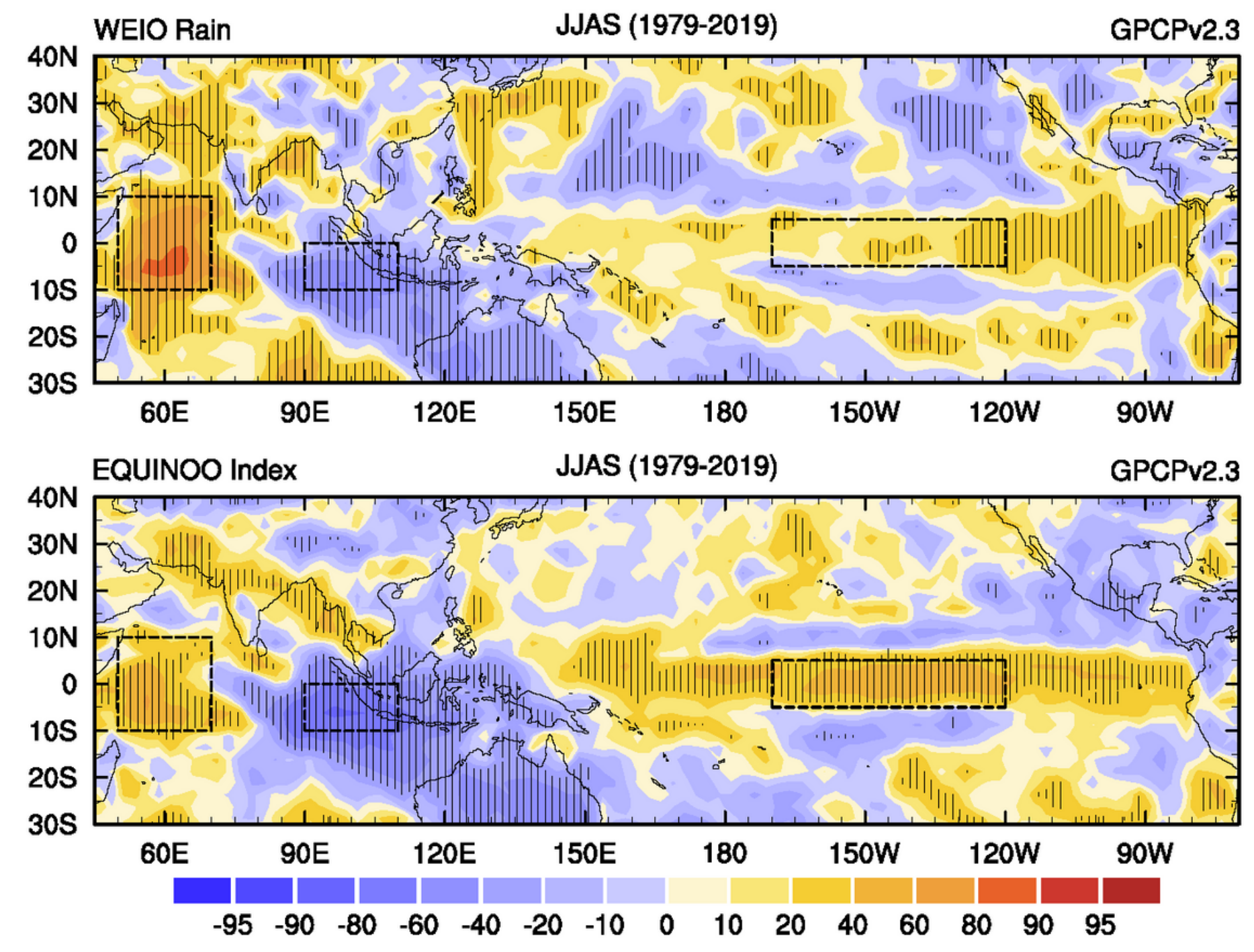

Figure 12

Distribution of correlation coefficient of a) WEIO rainfall versus rainfall at every grid over the tropical AsiaPacific sector (top panel) and b) EQUINOO index versus rainfall at every grid over the tropical Asia-Pacific sector (bottom panel). Black dashed boxes show regions of western and eastern equatorial Indian Ocean (WEIO and EEIO respectively) and equatorial central Pacific (NINO3.4). Correlations significant at $95 \%$ are stippled. 

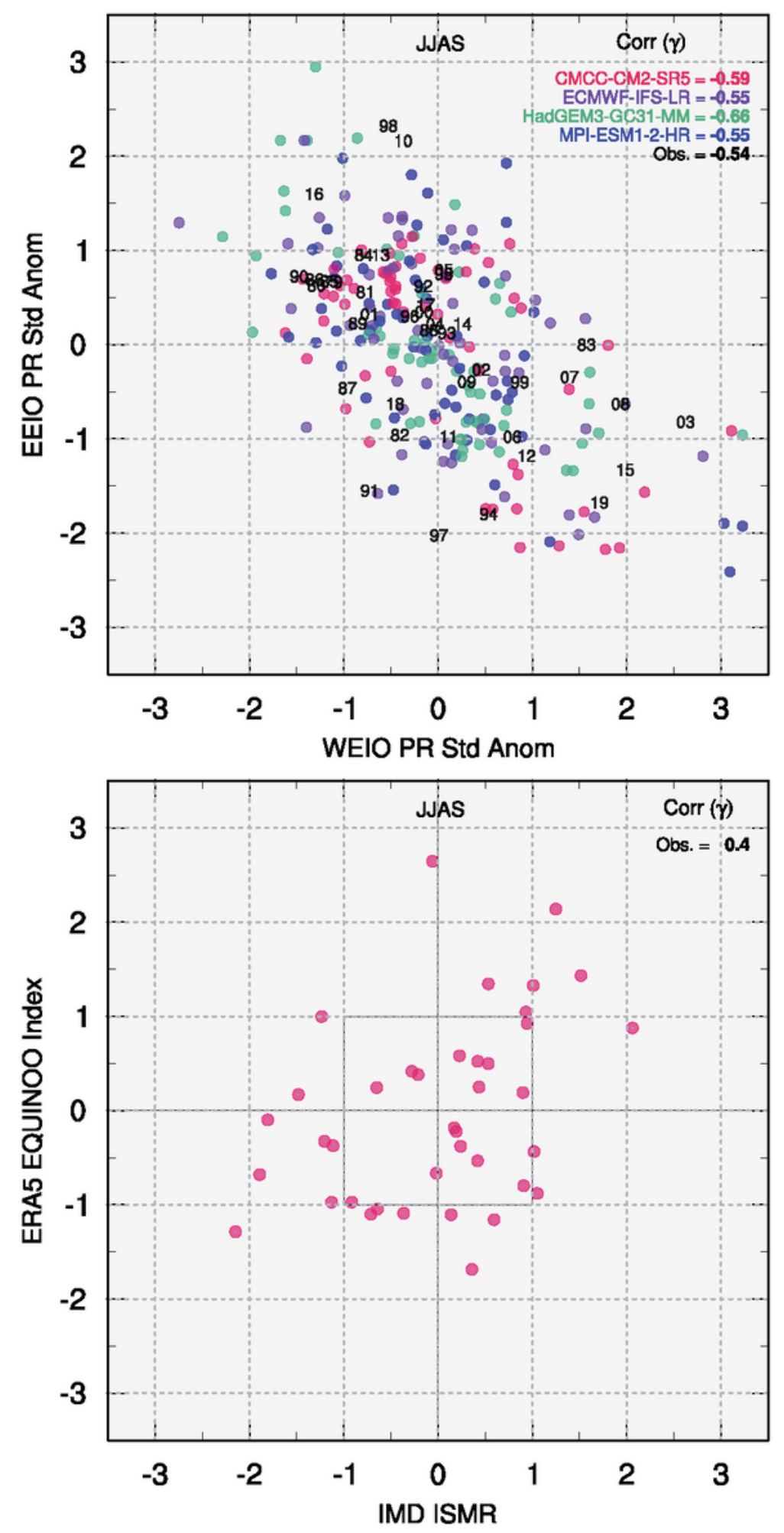

\section{Figure 13}

Scatter plot of standardized anomalies of a) EEIO rainfall versus WEIO rainfall from observation (GPCP rainfall denoted by the last two digits of the years) and 4 representative models of CMIP6 Grp (top panel). b) Scatter plot of standardised anomalies of ISMR versus EQUINOO index from observation (IMD rainfall and ERA-5 data). The corresponding correlations are given in top-right corner. 

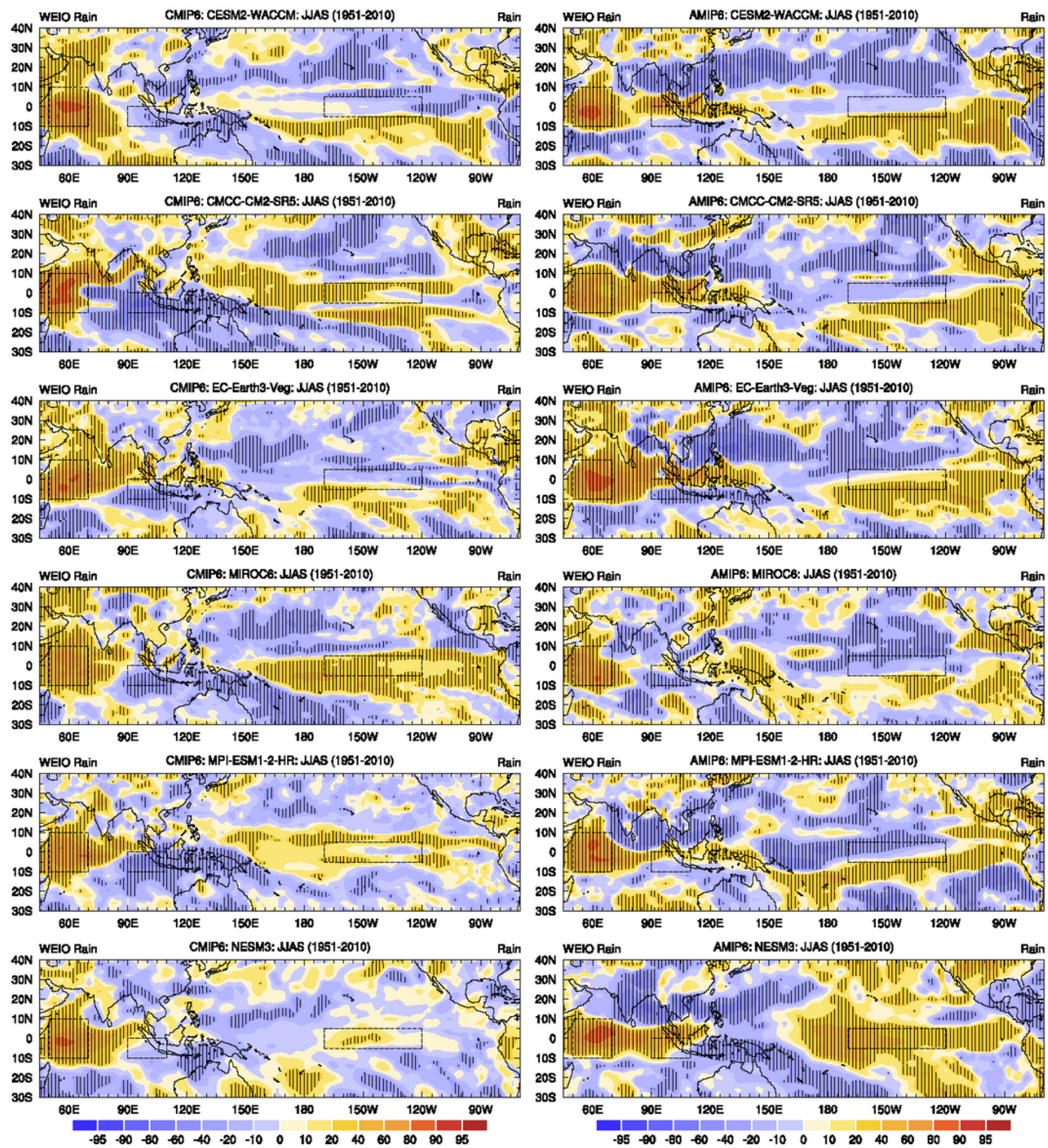

\section{Figure 14}

Distribution of correlation coefficient of WEIO rainfall versus rainfall at every grid over the Asia-Pacific region from coupled (left panels) and atmosphere-only (right panels) simulations of a) CESM2-WACCM , b) CMCCCM2-SR5, c) EC-Earth3-Veg, d) MIROC6, e) MPI-ESM1-2-HR and f) NESM3. Black dashed boxes show regions of western and eastern equatorial Indian Ocean (WEIO and EEIO respectively) and equatorial central Pacific (NINO3.4). Correlations significant at 95\% are stippled. 


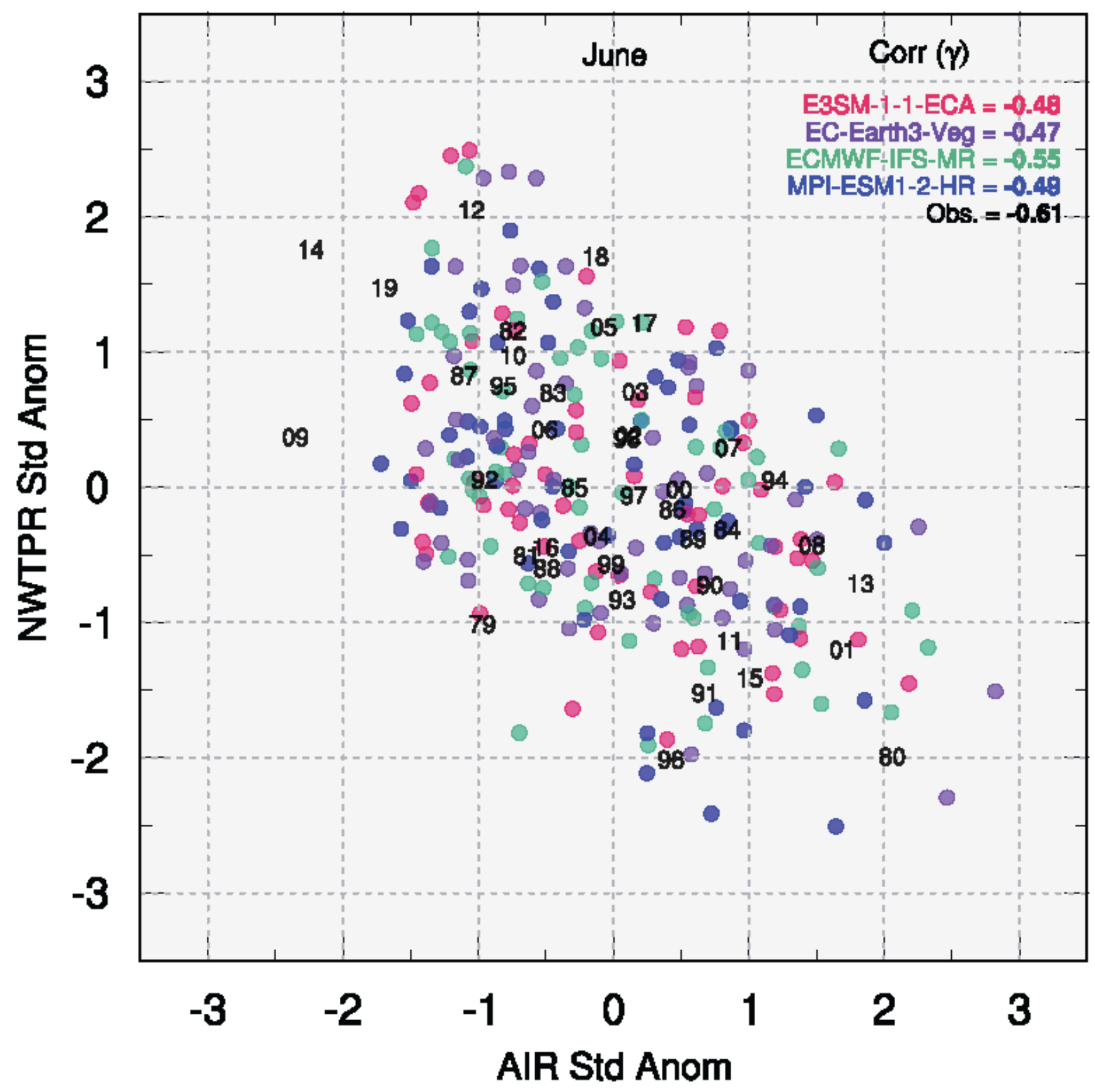

Figure 15

Scatter plot of standardized anomalies of June all-India rainfall (AIR) versus June NWTPR from observation (denoted by the last two digits of the years) and 4 representative models of CMIP6 Grp. The corresponding correlations are given in top-right corner. 